\title{
Automatic processing-based method for biometric characterization of biological images
}

Morgado F.*, Posada N.G.**, Chavez M.G.*, Soares A.M.V.M.* and Lopez M.A.G.**

* CESAM \& Departamento de Biologia, Universidade de Aveiro, Campus Universitário de Santiago, 3810193 Aveiro, Portugal

** Departamento de Electrónica, Universidade de Aveiro, Campus Universitário de Santiago, 3810-193 Aveiro, Portugal

Email: fmorgado@ua.pt

Within the framework of the morphological analysis of species, biometry has its starting point in the information obtained from measurable (discrete or continuous) specimens' features. Biometry allows establishing useful relationships for the interpretation of the forms expressed either graphically or by applying statistical analyses of a higher degree of complexity. In that sense, computer-aided methods for morphological analysis have been developed and reported. Geometric morphometrics is the statistical analysis of shape variation and its covariation with other variables, representing a new paradigm in the study of variation and covariation of the shape of biological structures. This work proposes a new fully automatic method for biometric characterization of biological images. This method combines properly several image processing-based techniques (Fig. 1), which have been organized in four well-defined steps: (1) Preprocessing - to enhance image details and landmarks; (2) Segmentation - to accurately detect the contour and computing elliptical axis (diameters) of organisms under study; (3) Object tracking - detecting and counting gonadic cells masses; and (4) Feature extraction - to measure selected biometric parameters (area, perimeter, maximum and minimum diameters, eccentricity, solidity and intensity-based statistics among others). A method prototype was implemented in MATLAB (R2013a). Figure 2 show a complete running example of the developed prototype. The method allows geometric assessment of images morphological variations throughout visual features such as shape, color and texture using a set of (200) random selected planktonic organisms images to develop a application to different biological domains such as taxonomy, phylogeny, evolution, biogeography, ecology and behavior. Visual features based on image content descriptors proved to help in the diagnostic of different biological processes and can be also used to find images of interest and to retrieve relevant information for clinical cases.

[1] Rodenacker K. and Bengtsson E., Analytical Cellular Pathology, 25, 1-36, 2003.

[2] Lopez M.A.G et al., Microscopy and Microanalysis, 19 (Suppl. 4), 75-76 2013.

[3] Adams, D. C. et al., Hystrix, the Italian Journal of Mammalogy, 24, 7-14, 2013. 
(1) Pre-processing

(2) Segmentation

(3) Object Tracking

(4) Feature Extraction
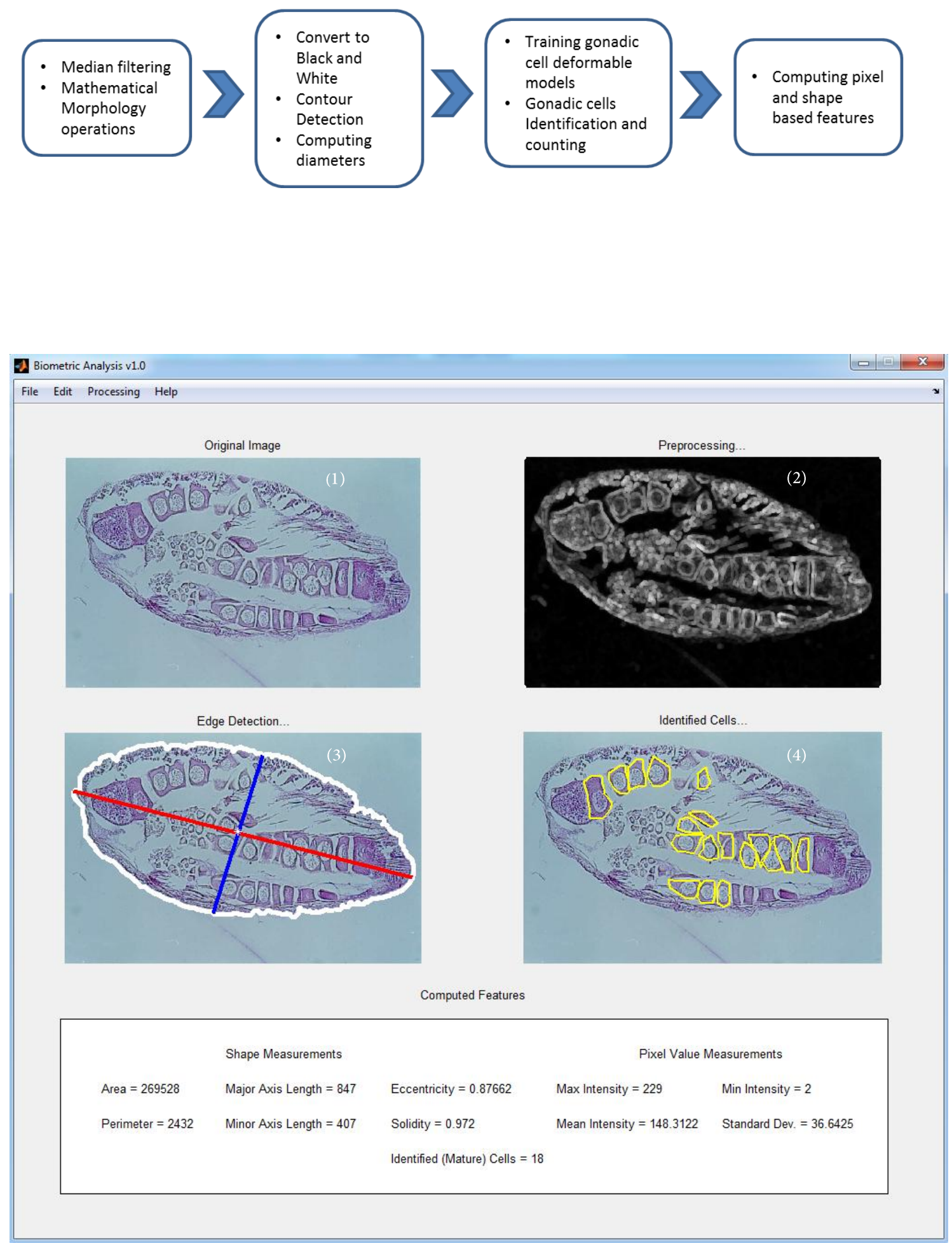

Fig.2. A full running example of the developed prototype: (1) Original image, (2) Preprocessing; (2) Resulting segmented organism (detected contour and diameters); (4) Recognized mature cells; and (5) Computed features. 